\title{
Optimal Control of Phosphorus Pollution in Lake Taihu with Wind-induced Nonlinear Feedbacks
}

\author{
Gael Bonnardot \\ Antai School of Economics and Management, \\ Shanghai Jiaotong University, \\ 200030 Shanghai, China \\ Gael.Bonnardot@eleves.ec-nantes.fr
}

\begin{abstract}
We propose a new model of transboundary pollution for Lake Taihu in China. This model takes into account the re-suspension of phosphorus, the main pollutant in shallow lake eutrophication. Wind is identified as the main cause for phophourus re-suspension, thus wind speed, modeled as a stochastic variable, is introduced in pollutant dynamics. Moreover, asymmetry between main polluters, namely Jiangsu and Zhejiang provinces, is considered. Model parameters are estimated based on recent ecological and economic studies of Lake Taihu and its region. Thanks to a technique introduced by Judd et al. (2011) for economic models, we compute the numerical solution of this differential game with stochastic non-linear dynamics. As a result we find the optimal pollution loadings for both provinces. Loading profiles are coherent with intuition: they decrease with pollution concentration and wind speed. Cumulative loadings of both regions are compared with the symmetric case. Finally, we find that in the case of Lake Taihu, policy makers should curve Zhejiang's incentive to emit by placing a tax on agricultural activities. Revenues generated from those taxes could fund sustainable development policies, thus improving pollution abatement for Lake Taihu.
\end{abstract}

Keywords: Differential Game, Eutrophication, Asymmetry, Stochastic Dynamics, Feedback Nash Equilibrium

\section{INTRODUCTION}

Ever since China's opening to trade and industrial development in the 1980s water eutrophication in most lakes has been growing at the pace of the national economy. The activity near Lake Taihu, the country's third largest lake, contributes now to more than $10 \%$ of China's GDP. But pollution abatement in this region has been neglected during several years. To the point that in 2007 a massive Microcystis bloom overwhelmed a waterworks that supplies Wuxi city on Lake Taihu's northern shore, leaving more than 2 million people without drinking water for a week. Qin et al. [1] describe this crisis and its causes. Among the factors which contributed to such a disaster are nonpoint pollution sources, namely, nutrients released from floor deposits and waste discharge from agricultural fields. These are the two main sources of nitrogen and phosphorus responsible for the algal blooms.
This is a typical case of a transboundary pollution problem. A great majority of these problems have been approached in the past decades from the game theoretical angle; Missfeldt gives a complete review of the fundamental contributions in this field in [2]. For instance, Zhao et al. [3] formulated the case of Lake Taihu as a discrete time dynamic game by. The authors use KKT conditions and a simple algorithm to obtain a model with transfer tax. However, their model does not capture the structural time dependence exhibited by the main pollutant, phosphorus. To capture this time dependence we use a differential game.

There has been an extensive use of differential games in the literature of transboundary pollution problems. For a complete review see Zaccour and Jorgensen [4]. Here we will merely highlight the contribution of those modeled eutrophication dynamics. Bayramoglu [5] considers the eutrophication of the Black Sea, mainly caused by Romania and Ukraine. The author explicitly takes into account fishing and agricultural activities in the utilities of both countries and compares the outcome of cooperative and non-cooperative strategies. The state dynamics are linear, omitting phosphorus re-suspension all together. Mäler, Xepapadeas and De Zeeuw [6] sets important foundations for future research on dynamics and control of shallow lakes. Assuming a particular function in describing internal pollution feedbacks, they demonstrate that two steady states are possible, one in the oligotrophic zone and one in the eutrophic one. Depending on the model parameters both states are reachable, and in some cases, only the initial value of the state can influence the outcome, good or bad. This particularity, called a Skiba point, is studied more in detail by Wagener [7] for the shallow lake system; Kossioris et al. [8] enlarges the framework set in [6] and propose a numerical solution for the Feedback Nash equilibrium, a problem too complicated to be solved analytically. Few papers study optimum pollution control for a given lake. Hein [9] studied the case of Lake De Wieden in the Netherlands, non-linear state dynamics are constructed according to observations made of phosphorus dynamics in this lake. 
Following this attempt to find a best fit between dynamic models and reality on the field, we base ourselves on ecological research on Lake Taihu in order to model pollution dynamics in this lake. Throug a synthetic work on Lake Taihu provided by Qin et al. [10] it is apparent that wind plays an important role in the evolution of the main polluting indicator: total phosphorus concentrations. Therefore, we will take into account wind speed in total phosphorus dynamics. Because the two main polluting agents are provinces which have different economic ties with Lake Taihu, we will introduce asymmetry between the players and will compare the results with the symmetric case. Symmetry is assumed in the great majority of papers on this subject, and yet there are obvious differences between players in terms of benefits and costs of pollution. Though asymmetry has been explored (e.g. List and Mason [11], in the case of transboundary pollution between federal states in the United States), to the authors best knowledge, it has not yet been taken into account in the case of lake eutrophication.

\section{THE MODEL}

\section{1) Province Utilities}

We consider $n=2$ economic agents in our model who take actions $a_{i}, i \in[1,2]$. Actions are expressed in terms of total phosphorus concentration in the lake. The provinces produce essentially agricultural goods which, due to non-point pollution, increase total phosphorus concentrations in Lake Taihu: $P(t)$. The latter is shared by both agents and its state is characterized by $P(t)$.

The range of actions for province $i$ is given by $a_{i}(t) \in\left[0, A_{i}\right]$. Without loss of generality we can suppose that $A_{1}=A$, and $A_{2}=\alpha A$, where $A, \alpha>0$. This is to say that one province has bigger production, i.e polluting power than the other province. The amount of phosphorus loaded in Lake Taihu, though environmentally damageable, is directly linked to each province's benefits, expressed by a strictly increasing and concave function:

$$
\Pi_{i}\left(a_{i}\right)=a_{i} A_{i}-a_{i}^{2} / 2
$$

The stock of phosphorus $P$ is responsible for the eutrophication of the lake for which each province incurs a cost $C_{i}$. Following Dragone et al. (2010), we model each province's costs by strictly increasing convex functions. Because the two provinces are not symmetric, the cost due to the eutrophication varies according to the province:

$$
C_{i}=D_{i}[P(t)]^{2} / 2
$$

Thus, the flow of net benefits at any point in time for agent I can be expressed as: $\Pi_{i}\left(a_{i}\right)-C_{i}(P)$. The latter will serve as province's $i$ utility function.

$$
U_{i}\left(a_{i}, P\right)=a_{i} A_{i}-a_{i}{ }^{2} / 2-D_{i}[P(t)]^{2} / 2
$$

\section{2) Phosphorus Dynamics}

For Lake Taihu, loadings and other polluting actions contribute to the variation of total phosphorus concentration in the lake at time $t, P(t)$, which evolves according to the following non-linear equation of motion:

$$
\dot{P}(t)=a_{1}(t)+a_{2}(t)-k P(t)+f(P(t))
$$

In equation (4) the second term $-k P(t)$ stands for the

TABLE I. WIND-SPEED INFLUENCE ON TP RELEASE

\begin{tabular}{cccr}
\hline Wind & Frequency & Speed & Release of TP \\
\hline Calm & $12 \%$ & $w<2 \mathrm{~m}^{\mathrm{s}} \mathrm{s}^{-1}$ & $108 \mathrm{t} / \mathrm{year}$ \\
Gentle & $82 \%$ & $2<w<6 \mathrm{~m} \cdot \mathrm{s}^{-1}$ & $5.69 .10^{-4} \mathrm{mg} / \mathrm{m}^{2} . \mathrm{s}$ \\
Gusty & $6 \%$ & $6 \mathrm{~m} \cdot \mathrm{s}^{-1}<w$ & $2.14 .10^{-3} \mathrm{mg} / \mathrm{m}^{2} . \mathrm{s}$ \\
\hline
\end{tabular}

standard linear degradation of phosphorus, this degradation can be explained by plant absorption, sedimentation adsorption or chemical interactions with other chemical species in the lake. The third term $f(P(t))$ describes the non-linear internal feedbacks explained just above. According to [12], wind has an important influence on phosphorus re-suspension and thus total phosphorus concentrations in Lake Taihu,. Table 2. describes the characteristics associated with three types of wind the research team has identified.

We introduce a new time-dependent state $w(t)$, representing for every $t$ the main speed of the wind blowing across the lake. Based on a wind-induced internal loading model described in [13], we assume internal feedbacks to be a fraction of phosphorus concentration proportional to wind speed. Thus, for Lake Taihu internal feedbacks are modeled as:

$$
f(P(t))=\zeta \cdot w(t) \frac{P(t)}{P(t)+1}
$$

Like any phenomenon linked to weather fluctuation wind speed is likened to a stochastic variable. Moreover it is positive at all time. There are a number of ways to take these two conditions into account and describe $w(t)$ for all $t$. We have chosen the following:

$$
\dot{w}(t)=w(t)(\exp (\epsilon(t))-1)
$$

Where $\epsilon(t) \sim \mathcal{N}\left(0, \sigma^{2}\right), w(0) \geq 0$. Thanks to data provided by the Taihu research center, compiled in [10], we estimate $\sigma=0.20$.

\section{THE SHALLOW LAKE GAME}

Each province has the incentive to choose a strategy $a_{i}$ that will maximize the present value of its net benefits over an infinite time horizon, subject to both phosphorus and wind dynamics:

$$
\max _{a_{i}} E\left(\int_{0}^{\infty} e^{-\rho t} U_{i}\left(a_{i}(t), P(t)\right) d t\right)
$$

$$
\begin{gathered}
\text { st. } \quad \dot{P}(t)=a_{1}(t)+a_{2}(t)-k P(t)+\zeta \cdot w \frac{P(t)}{P(t)+1} \\
\dot{w}(t)=w(t)(\exp (\epsilon(t))-1), \epsilon(t) \sim \mathcal{N}\left(0, \sigma^{2}\right)
\end{gathered}
$$


This problem cannot be solved analytically. However it is possible to calculate a numerical solution. To solve equations (7)-(9) we use a technique described in [14]. The first step consists in shifting to a discrete model:

$$
\begin{gathered}
\max _{a_{i t}, P_{t+1}=0, . \infty} E_{0} \sum_{t=0}^{\infty} \beta^{t} U_{i}\left(a_{i t}, P_{t}\right) \\
\text { st. } \quad P_{t+1}=a_{1 t}+a_{2 t}+(1-k) P_{t}+\zeta \cdot w \frac{P_{t}}{P_{t}+1} \\
\quad \ln w_{t+1}=\ln w_{t}+\epsilon_{t+1}, \epsilon_{t+1} \sim \mathcal{N}\left(0, \sigma^{2}\right)
\end{gathered}
$$

Where the initial condition $\left(P_{0}, w_{0}\right)$ is given. $E_{t}$ is the expectation operator conditional on information at time $t$. $a_{t}, P_{t}$ and $w_{t}$ are phosphorus concentration induced by loadings, phosphorus concentration and wind speed respectively. $\beta=e^{-\rho}$ in equation (10) is the discount factor of the discrete model. We solve this problem using dynamic programming, we introduce the value functions $V_{i}(P, w)$ which verify the associated Bellman equation:

$$
\begin{gathered}
V_{i}(P, w)=\max _{P^{\prime}, a}\left\{U_{i}\left(a_{i}, P\right)+\beta E\left[V_{i}\left(P^{\prime}, w^{\prime}\right)\right]\right\} \\
\text { s.t. } P^{\prime}=a_{1}+a_{2}+(1-k) P+\zeta \cdot w \frac{P}{P+1} \\
\ln w^{\prime}=\ln w+\epsilon^{\prime}, \epsilon^{\prime} \sim \mathcal{N}\left(0, \sigma^{2}\right)
\end{gathered}
$$

The prime on variables stands for next-period values, and $E\left[V\left(P^{\prime}, w^{\prime}\right)\right] \equiv E\left[V\left(P^{\prime}, w^{\prime}\right) \mid P, w\right] \quad$ is the expectation of $V\left(P^{\prime}, w^{\prime}\right)$ conditional on the state $(P, w)$. The next step is to approximate $\mathrm{V}$ with a parametric function, for example a complete ordinary polynomial of the type:

$$
Q(P, w ; b)=\sum_{k=0}^{L} \sum_{j=0}^{k} b_{j}\left(\begin{array}{l}
k \\
j
\end{array}\right) w^{j} P^{k-j}
$$

Where $b=\left(b_{0}, b_{1}, \ldots, b_{n}\right) \in R^{n+1}$ is a vector of polynomial coefficients, and $\mathrm{L}$ is the degree of the polynomial.

Since $P^{\prime} \quad$ is known at present and $w^{\prime}=w \exp \left(\epsilon^{\prime}\right)$, the conditional expectation of $Q\left(P^{\prime}, w^{\prime} ; b\right)$ is as follows:

$$
\begin{aligned}
E\left[Q\left(P^{\prime}, w^{\prime} ; b\right)\right] & =E\left[b_{0}+b_{1} P^{\prime} \ldots+b_{n} w^{L} \exp \left(L \epsilon^{\prime}\right)\right] \\
& =b_{0}+b_{1} P^{\prime} \ldots+b_{n} w^{L} E\left[\exp \left(L \epsilon^{\prime}\right)\right] \\
& =b_{0} I_{0}+b_{1} I_{1} P^{\prime}+\cdots+b_{n} I_{n} w^{L} \\
& =\gamma_{0}+\gamma_{1} P^{\prime}+\cdots+\gamma_{n} w^{L}=Q\left(P^{\prime}, w ; \gamma\right)
\end{aligned}
$$

We note $e_{l}$ is the $l$-th moment of the function $\exp \left(\epsilon^{\prime}\right)$, $l=0,1, \ldots, L$. Since $\epsilon^{\prime} \sim \mathcal{N}\left(0, \sigma^{2}\right)$ the moments are written:

$$
e_{l}=\frac{1}{\sqrt{2 \pi \sigma}} \int_{-\infty}^{+\infty} \exp \left(l \epsilon^{\prime}\right) \exp \left(-\frac{\left(\epsilon^{\prime}\right)^{2}}{2 \sigma^{2}}\right) d \epsilon^{\prime}
$$

The $I_{j}$ are the defined for all $j$ belonging to $[|1, n|]$, by

$$
\left\{I_{1}, I_{2}, I_{3}, I_{4}, I_{5}, I_{6}, \ldots, I_{n}\right\}=\left\{1,1, e_{1}, 1, e_{1}, e_{2}, \ldots, e_{L}\right\}
$$

And finally we define vector $\gamma$ by $\gamma_{j}=b_{j} I_{j}$, for all $j$.

From (17) we conclude that the expectation of polynomial function $Q$ is given by the same polynomial function evaluated at a different coefficients vector $\gamma$. From these calculations we deduce that if we approximate region $i$ 's true value function $V_{i}(P, w)$ with a polynomial function $\widehat{V}_{i}\left(P, w ; b_{i}\right)$ of the form (16) then, by means of $(17)$, we can rewrite the Bellman equation as:

$$
\begin{gathered}
\widehat{V}_{i}\left(P, w ; b_{i}\right) \cong \max _{P^{\prime}, a}\left\{U_{i}\left(a_{i}, P\right)+\beta \widehat{V}_{i}\left(P^{\prime}, w ; \gamma_{i}\right)\right\} \\
\text { s.t. } P^{\prime}=a_{1}+a_{2}+(1-k) P+\zeta \cdot w \frac{P}{P+1} \\
\ln w^{\prime}=\ln w+\epsilon^{\prime}, \epsilon^{\prime} \sim \mathcal{N}\left(0, \sigma^{2}\right) \\
\gamma_{i, j}=b_{i, j} I_{j}, j=1 \ldots n, i=1,2
\end{gathered}
$$

In this transformed version of the Bellman equation the stochastic characteristic is contained in the moments $e_{l}$ and thus in the integrals $I_{j}$. To solve this problem there are basically two steps:

First, pre-compute the integrals $I_{j}$ using (18). Then find the two vectors $b_{1}$ and $b_{2}$ that solve equations (19)(22). Both players take turns in choosing a loading quantity according to the other one's own loading choice. This way, in average, both provinces have the same knowledge on the other provinces choice of optimal loadings given the value of pollution and wind speed at time $t$. The algorithm thus provides us with an approximate solution to the Feedback Nash Equilibrium.

\section{COMPUTATIONAL RESULTS}

\section{1) Two player profiles}

Thanks to statistical yearbooks of both Jiangsu and Zhejiang provinces, [15], [16], which provide data on agricultural activity (Figure 1) we estimate the two asymmetry parameters. There are mainly three pollution sources: the local population (Pop), field agriculture (Agri) and livestock (Live). Jiangsu and Zhejiang are set to be respectively players 1 and 2 . Using economic data and profits made per year we find that $\alpha=0.68$ and $\mu=0.5$ This means that Zhejiang incurs two times less damage from pollution but its maximum production and pollution capacity only amounts to $68 \%$ of that of Jiangsu. The program is run on a $2 \mathrm{GHz}$ Dual Core laptop with MATLAB R2012a. We chose for the degree of the polynomial $L=3$, and the states $P$ and $w$ vary in $[0.01,1.2] \times[0.1,12]$, each interval split into 80 discrete values. We obtain figure 2 . 


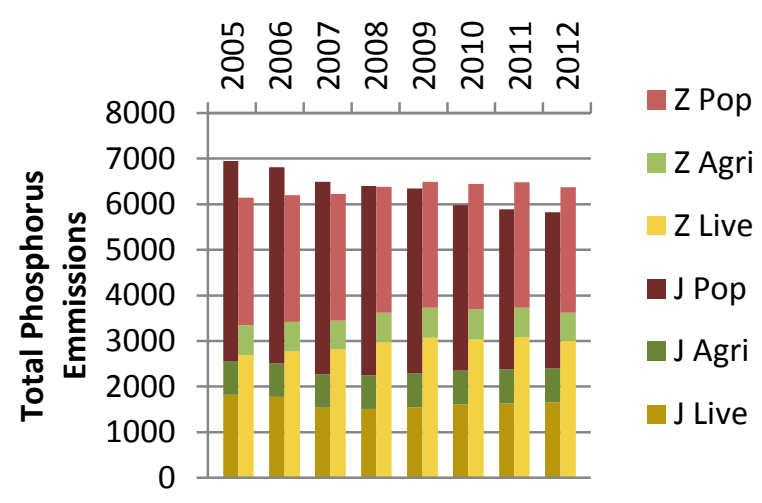

Figure 1. Phosphorus Emissions of Jiangsu (J) and Zhejiang (Z)

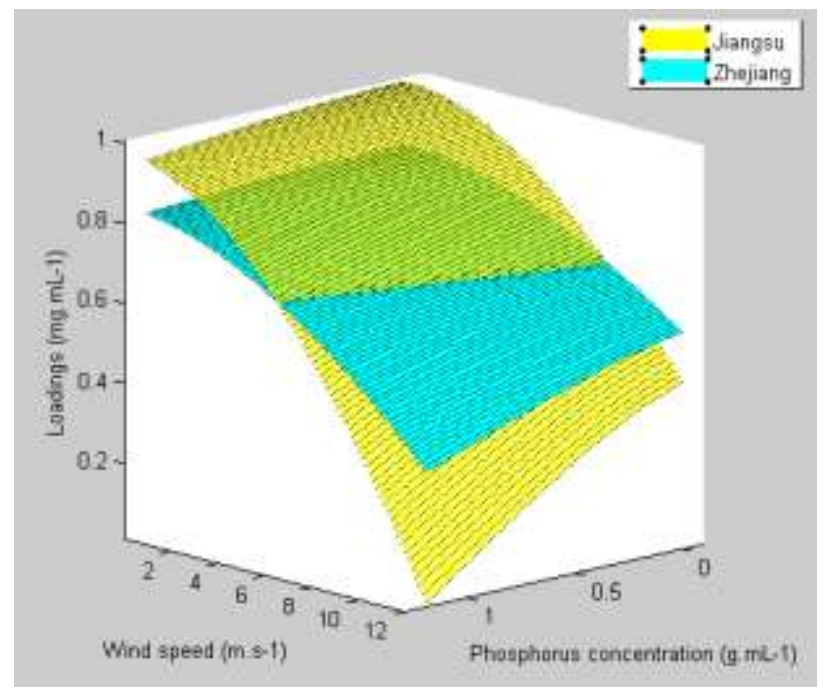

Figure 2. Jiangsu and Zhejiang optimal loadings

First of all we observe that the faster the wind and the higher the phosphorus concentration the lower the loadings. This is coherent with each provinces incentive not to pollute too much Lake Taihu because of the costs both regions incur from pollution. Second, it appears that when pollution and wind speed is high Jiangsu will tend to emit less than Zhejiang. This is due to the fact that costs for Jiangsu regarding pollution are higher, thus Jiangsu has less incentive to emit than Zhejiang. In the last 6 to 10 years, and still now, Lake Taihu is in an eutrophied state and total phosphorus concentrations are around 0.08 g. $\mathrm{mL}^{-1}$. In average the wind speed is $4 \mathrm{~m} . \mathrm{s}^{-1}$. Therefore during a whole year Zhejiang emissions are in average higher than those of Jiangsu. We verify that the model results are coherent with our estimations of recent nonpoint agricultural phosphorus emissions of the two provinces (figure 1.).

\section{2) Comparison with the symmetric model}

Figure 3. shows the difference in cumulative loadings between the asymmetric and the symmetric case. It must be specified that in the considered case of symmetry we set $\mu=1$ and $\alpha=1$, so that both regions are supposed to have the same characteristics as Jiangsu. We see that if the assumption of symmetry is made there is a big gap between supposed loadings and actual loadings. Moreover this gap grows bigger with pollution levels and wind speed.

Furthermore, since cost of pollution in comparison with polluting capacity is the critical factor and we can estimate the polluting capacity of Zhejiang the policy maker must increase $\mu$ order to curb the incentive for Zhejiang to emit pollution. This 'increase' in $\mu$ could be achieved by increasing the tax of pollution in Zhejiang province or the central government could penalize Zhejiang province if emission standards are not met.

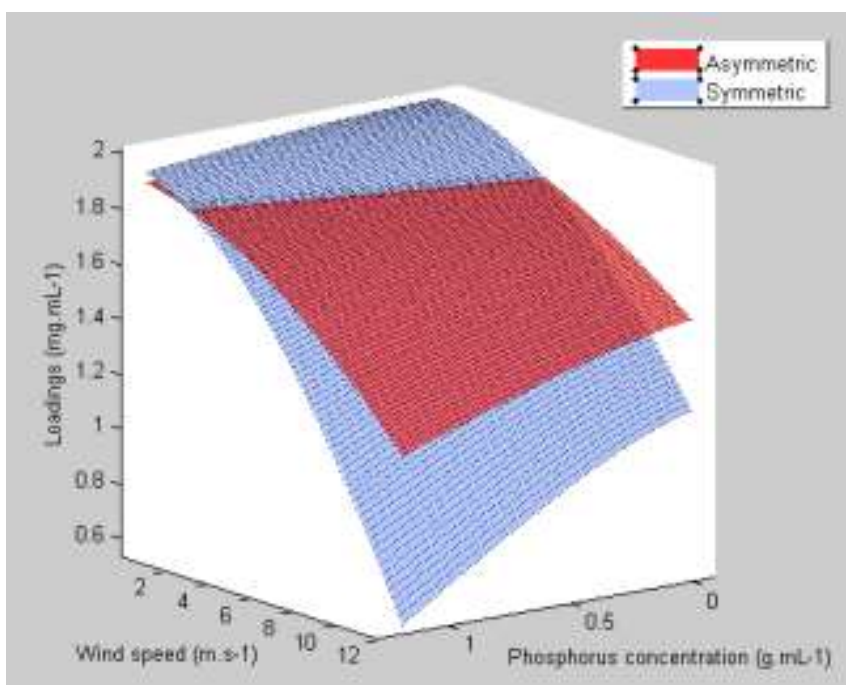

Figure 3 Cumulative optimal loadings

\section{CONCLUSION}

Pollution of Lake Taihu is modeled as a game of transboundary pollution between two main actors: Jiangsu and Zhejiang province. Thanks to studies both from the optimal control and ecological literature we have proposed a model for pollution dynamics in Lake Taihu. The model parameters are then calibrated given ecological data on Lake Taihu as well as economical data on Jiangsu and Zhejiang provinces. To solve this non-linear, stochastic differential game we base ourselves on a resolution method developed in [14]. We adapt this method to our model and program the algorithm on MATLAB. We solve the two-region asymmetric model given that each region take turns in observing the others loadings before choosing its own loading quantity. Since the periods are sufficiently small (6 hours) compared to the total time horizon (20 days) the solution gives us an approximation of the Feed-Back Nash Equilibrium. Following the computation of the FBNE solution, we study the specific case of Jiangsu and Zhejiang provinces according to economic data on both provinces provided by the yearly statistical books. We find that for high levels of pollution Zhejiang's pollution loadings are more likely to surpass those of Jiangsu. This conclusion is coherent with calculations of past emissions. Furthermore, we compare our outcome to one where the policy maker would suppose that the two regions would have the same 
emission capacity and cost of pollution as Jiangsu $(\alpha=\mu=1)$. We observe that cumulative loadings are significantly different, particularly when pollution levels in the lake are high. Therefore to improve pollution levels in Lake Taihu, policy makers should identify which region has smaller costs related to pollution levels in the lake (Zhejiang, according to our calculations) and penalize emissions in a way to balance with Jiangsu Province. The costs incurred to the region could be refunded in terms of pollution abatement subsidies and promote more environmental-friendly forms of agriculture. That way there is no incentive for Zhejiang to deviate from the pollution abatement plan.

\section{ACKNOWLEDGMENTS}

I would like to express my gratitude to my mentor Shoude Li for his help and support, as well as the educational staff of Shanghai Jiaotong University who encouraged me during my Masters and research.

\section{REFERENCES}

[1] B. Qin et al., 2010. A drinking water crisis in Lake Taihu, China: linkage to climatic variability and lake management. Environmental Management 45, pp 105-112.

[2] F. Missfeldt, 1999. Game theoretic modelling of transboundary pollution. Journal of Economic Surveys 13.

[3] L. Zhao et al., 2013. Harmonizing model with transfer tax on water pollution across regional boundaries in a China's lake basin. European Journal of Operational Research 225, pp 377-382.

[4] G. Zaccour, S. Jorgensen, G. Martin-Herran, 2010. Dynamic Games in the economics and management of pollution. Environ Model Assess 15,pp 433-467.

[5] B. Bayramoglu, 2006. Transboundary pollution in the Black Sea: comparison of institutional arrangements. Enbironmental \& Ressource Economics 35, pp 289-325.

[6] K.G Mäler, A. Xepapadeas, A. De Zeeuw, 2000. The economics of shallow lakes. Center for Economic Research 2000-69.

[7] F.O.O Wagener, 2003. Skiba points and heteroclinic bifurcations, with applications to the shallow lake system. Journal of Economic Dynamics \& Control 27, pp 1533-1561.

[8] G. Kossioris, M. Plexousakis, A. Xepapadeas, A. De Zeeuw, K.G Mäler, 2008. Feedback Nash equilibria for non-linear differential games in pollution control. Journal of Economic Dynamics \& Control 32, pp $1312-1331$.

[9] L. Hein, 2006. Cost-efficient eutrophication control in a shallow lake ecosystem subject to two steady states. Ecological Economics 59, pp 429-439.

[10] B. Qin et al., 2006. Estimation of internal nutrient release in large shallow Lake Taihu, China. Science in China Series D Earth Sciences 49, pp 38-50.

[11] J. List, M. Mason, 2001. Optimal institutional arrangements for transboundary pollutants in a second-best world: evidence from a differential game with asymmetric players. Journal of Environmental Economics and Management 42, pp 277-296.

[12] B. Qin et al., 2006. Estimation of internal nutrient release in large shallow Lake Taihu, China. Science in China Series D Earth Sciences 49, pp 38-50.

[13] B. De Jong, 2004. Generic models for phosphorus retention in shallow lakes. A multi-lake study. Thesis Aquatic Ecology and Water Quality 10.

[14] K.L. Judd, Maliar, S., Maliar, L., 2011. How to solve dynamic stochastic models computing expectations just once. NBER Working Paper 17418.

[15] 2013 Jiangsu Statistical Yearbook. China Statistic Press. Beijing Info Press.
[16] 2013 Zhejiang Statistical Yearbook. China Statistic Press. Beijing Info Press. 
